\title{
Combinatorial Library Screening for Developing an Improved- Split- Firefly Luciferase Fragment-Assisted Complementation System for Studying Protein-Protein Interactions
}

\author{
Ramasamy Paulmurugan and Sanjiv S. Gambhir \\ Departments of Radiology and Bioengineering, Bio-X Program Molecular Imaging Program at \\ Stanford (MIPS), Stanford University School of Medicine, James H. Clark Center, 318 Campus \\ Drive, East Wing, 1st Floor, Stanford, CA 94305-5427, USA
}

\begin{abstract}
Split reporter based bioluminescence imaging is a useful strategy for studying protein-protein as well as other intracellular interactions. We have used a combinatorial strategy to identify a novel split site for firefly luciferase with improved characteristics over previously published split sites. A combination of fragments with greater absolute signal with near zero background signals was achieved by screening 115 different combinations. The identified fragments were further characterized by using five different interacting protein partners and an intramolecular folding strategy. Cell culture studies and imaging in living mice was performed to validate the new split sites. In addition, the signal generated by the newly identified combination of fragments (Nfluc 398/Cfluc 394) was compared with different split luciferase fragments currently in use for studying protein-protein interactions and shown to be markedly superior with a lower selfcomplementation signal and equal or higher post-interaction absolute signal. This study also identified many different combinations of non-overlapping and overlapping firefly luciferase fragments that can be used for studying different cellular events such as sub cellular localization of proteins, cell-cell fusion, and evaluating cell delivery vehicles, in addition to protein-protein interactions, both in cells and small living animals.
\end{abstract}

\section{Keywords}

Molecular Imaging; Bioluminescence Imaging; Protein-Protein Interactions; Rapamycin; Synthetic Renilla Luciferase; Enhanced Green Fluorescent Protein

\section{Introduction}

Much of modern biological research is concerned with the how, when, and where of proteinprotein interaction. The need for simpler approaches to study these protein-protein interactions, particularly on a larger scale and especially in intact cells is great. Furthermore, if these interactions could be studied in intact living subjects this would allow additional insights into normal and diseased states. In order to understand such ubiquitous protein interactions, several techniques have been developed and are reviewed elsewhere ${ }^{1,2}$.

A protein fragment-assisted complementation (PFAC) strategy for studying protein-protein interaction involves the use of a combination of split reporter gene fragments that encode for split proteins that have relatively low affinity for each other and thus produce low signal.

Co-corresponding authors: Sanjiv Sam Gambhir/Ramasamy Paulmurugan, Stanford University School of Medicine, James H. Clark Center, 318 Campus Drive, 150 East Wing, 1st Floor, Stanford, CA 94305-5427, sgambhir@stanford.edu; paulmur8@stanford.edu. 
When these same fragments are fused to two interacting proteins of interest, the interaction of the two proteins drives complementation of the split reporters leading to a detectable signal. The PFAC strategy has been developed using a variety of reporter proteins including dihydrofolate reductase ${ }^{3,4}, \beta$-lactamase ${ }^{5}$, green fluorescent protein ${ }^{6}$, firefly luciferase ${ }^{7}$ and renilla luciferase ${ }^{8}$. Several of the currently available techniques for studying protein-protein interactions are restricted to using either cell lysates or intact cells. To extend these applications to small living animals, we and others have adopted a yeast two hybrid system 9,10 using bioluminescence. To develop a more robust and generalizable strategy for imaging protein interactions in living small animals we previously reported a split firefly luciferase fragment-assisted complementation and intein-mediated reconstitution system ${ }^{11}$. The fragment assisted complementation approach was subsequently also studied by others in small animals ${ }^{12}$.

The PFAC strategies based on split bioluminescent reporters (firefly luciferase and renilla luciferase) are particularly useful because of their applicability in imaging protein-protein interactions in intact cells and by direct extension to small living animals. In addition, these bioluminescent reporters hold potential advantages for use in small animals over other reporters particularly due to their low background signal ${ }^{13}$. This is in contrast to work with fluorescent reporters (e.g., green fluorescent protein, and red fluorescent protein) and reporters that use fluorescent substrates as a readout (e.g., $\beta$-lactamase and $\beta$-galactosidase) due to autofluorescence and confounding increases in background signal. $\mathrm{We}^{8,11}$ and others ${ }^{7}, 12,14$ have identified several combinations of split fragments for bioluminescent reporters such as firefly and renilla luciferase that are suitable for studying protein-protein interactions in living animals $8,11,12,14$. In addition, we also recently identified several combinations of firefly luciferase enzyme fragments that self-complement without assistance via protein-protein interactions ${ }^{15}$. These types of fragments are useful for studying cellular localization of tagged proteins, evaluation of cell macromolecular delivery vehicles, and also for studying cell-cell fusion. We have also recently applied split reporter strategies to study intramolecular folding of the estrogen receptor in intact cells and small living animals ${ }^{16}$.

The sensitivity of the split reporters in studying protein-protein interactions will in general depend on several variables including the affinity of the two interacting proteins of interest. The split firefly luciferase fragments used in our previous study showed a good sensitivity with the interacting protein partners $\mathrm{Id} / \mathrm{myoD}^{11}$, but they failed to produce significant levels of signal with the rapamycin mediated interacting proteins FRB/FKBP12. In the rapamycin mediated interaction strategy the small molecule rapamycin binds to the proteins FRB and FKBP12 and leads to the induction of both homo and heterodimerization between these proteins. This result contrasts with the combination of split firefly luciferase fragments utilized by others that are reported to have a greater level of signal with the rapamycin mediated FRB/FKBP12 interaction system but at a cost of increased background signal ${ }^{12}$. The renilla luciferase fragments (Nrluc 229 and Crluc 229) utilized in our previous study show significant levels of protein-protein interaction assisted luciferase signal for many different interacting partners with a near-zero background ${ }^{8,17}$. However, the wavelength of light emitted during the enzymatic reaction of renilla luciferase with its substrate coelenterazine is in the range $480-510 \mathrm{nM}$, and this wavelength range sometimes incurs limitations of light absorption by different proteins in biological tissues (e.g., hemoglobin) when extending this system to imaging studies in small living animals.

To improve the absolute signal by increasing the protein-protein interaction assisted luciferase signal, we also reported a fusion protein strategy where a single vector encodes both interacting partners and the split reporters separated by linker sequences as a fusion protein ${ }^{18}$. Whereas these published studies have already proven the utility of split 
bioluminescence reporters in studying protein-protein interactions, the focus of the current study was to use a combinatorial screening approach to identify new combinations of firefly luciferase fragments for studying protein-protein interactions with greater absolute signal and relatively low background that would be applicable to many different types of studies in cell culture and imaging small living animals.

We have collected over the last several years a library of N- and C- terminal fragments of firefly and renilla luciferase split reporter protein fragments in the course of developing and validating various split reporter strategies. In the current study, by applying a combinatorial screening approach to this library, we screened several combinations of $\mathrm{N}$ - and $\mathrm{C}$ - terminal firefly luciferase fragments with the interacting proteins FRB/FKBP12 and identified a new set of Nfluc and Cfluc fragments that have ideal properties of low background signal (low self-complementation) and a high signal after protein-protein interaction assisted complementation. In addition, this strategy also identified a number of combinations with and without overlapping regions and with and without self-complementing properties, in a single step. The selected combinations that produced the highest level of rapamycin induced luciferase signal were further evaluated with five other interacting protein combinations. An intramolecular folding strategy with the estrogen receptor and various ligands was also studied in cells and in mice with the identified optimal split reporters. The novel split reporters developed in this study will potentially increase the sensitivity and the generalizability of fragment-assisted complementation systems for studying protein-protein and other interactions in cells and small living animals.

\section{Materials and Methods}

\section{Chemicals, Enzymes and Reagents}

Restriction and modification enzymes, and ligase were obtained from New England Biolabs (Beverly, MA). TripleMaster Taq DNA polymerase was obtained from Brinkmann Eppendorf (Hamburg, Germany). All the constructs were made with a pcDNA3.1 (+) backbone. PCR was used for constructing all the vectors with different fragments and different interacting proteins. Rapamycin and all the different ER ligands used in this study were obtained from Sigma (St. Louis, MO). Lipofectamine transfection reagent was from Invitrogen (Carlsbad, CA 92008). The plasmid extraction kit and DNA gel extraction kit were from Qiagen (Valencia, CA). Coelenterazine was from Nanolight (Pinetop, AZ). Luciferin is from Xenogen (Alameda, CA). Bacterial culture media were from BD Diagnostic Systems (Sparks, MD). All cell culture media, fetal bovine serum, the antibiotics streptomycin, and penicillin, were from Invitrogen (Carlsbad, CA). The charcoal treated fetal bovine serum was from HyClone (Logan, UT). Oligonucleotide synthesis and DNA sequencing were performed by the Stanford Protein and Nucleic Acid facility.

\section{Construction of Plasmids}

All PCR amplified reporter fragments with corresponding restriction enzyme sites were inserted in the plasmids by replacing the Nrluc and Crluc portions of the vectors pcDNANrluc-FRB and pcDNA-FKBP12-Crluc used in our previous study ${ }^{17}$. Similarly the vectors with the interacting protein partners Id/myoD, HIF1- $\alpha / \mathrm{pVHL}, \mathrm{ER} / \mathrm{ER}$ and TK/TK were constructed by replacing the FRB and FKBP12 portions of the vectors. The ER intramolecular folding vector was constructed by first generating the vector pcDNA-NflucCfluc, followed by insertion of PCR amplified ER (amino acids 281-595) with BamHI restriction enzyme sites on either side. The digested dephosphorylated vector was used for inserting the Bam HI digested ER fragment and constructed pcDNA-Nfluc-hER-Cfluc. The orientations of the insert were initially confirmed by activity assay and further by sequencing. 


\section{Cell culture}

Human 293T embryonic kidney cancer cells (ATCC, Manassas, VA) and MCF7 human breast cancer cells were grown in MEM supplemented with 10\% FBS and 1\% penicillin/ streptomycin. MDA-MB-231 breast cancer cells and RL95 human uterine carcinoma cells were grown in DMEM-high glucose supplemented with 10\% FBS and 1\% penicillin/ streptomycin. For the experiments with estrogen receptors, we used the cells grown in medium with activated charcoal treated serum. The cells were grown at $37^{\circ} \mathrm{C}$ with $5 \% \mathrm{CO}_{2}$.

\section{Cell transfection and luciferase assay}

Transfections were performed in $24 \mathrm{~h}$ old cultures of 293T, MCF7 and RL95 ( 80\% confluent) cells. For transfections and co-transfection, $200 \mathrm{ng}$ or $200 \mathrm{ng}$ of each/well of DNA were used in 12 well culture plates. Lipofectamine transfection reagent was used as recommended by the manufacturer. For cell culture heterodimerization experiments, $40 \mathrm{nM}$ Rapamycin was added immediately after transfection. Similarly for ER ligand induced folding and homodimerization studies, $1 \mu \mathrm{M}$ of each ligand dissolved in DMSO was added immediately after transfection. DMSO was used as solvent control. Cells were assayed for luciferase activity by using LARII (Promega) assay reagent and for Renilla luciferase activity as by the method previously published ${ }^{19}$. Light measurements were performed in Turner Designs, 20/20 luminometer (Sunnyvale, CA) for $10 \mathrm{~s}$. Bio Rad protein assay reagent was used for measuring the protein concentrations in the cell lysates. The luciferase activities are represented as relative light units (RLU) per microgram of protein.

\section{Combinatorial screening by optical CCD camera imaging}

To screen the combinations of fragments, 50,000 cells (293T) plated in 96 well plates were transfected with different combinations of plasmids. For transfection $12.5 \mathrm{ng}$ of each plasmids/well were used. The cells were exposed to $40 \mathrm{nM}$ rapamycin immediately after transfection and imaged after $48 \mathrm{hrs}$ by adding $1 \mu \mathrm{g} /$ well D-Luciferin in $25 \mu \mathrm{l}$ of PBS with 1minute acquisition. For each sample one well served as control and another well with rapamycin. The plates were analyzed for rapamycin-mediated complementation of luciferase enzyme fragments.

\section{Optical CCD imaging of ligand induced intramolecular folding of the estrogen receptor in living mice}

All animal handling was performed in accordance with Stanford University Animal Research Committee guidelines. For imaging in living nude mice (nu/nu), 293T and RL95 cells stably expressing sensors 2 and 3 (Supplementary Figure S3) were used. Mice were anesthetized by i.p. injection of $\approx 40 \mu \mathrm{l}$ of a ketamine and xylazine (4:1) solution, and five millions cells of each stable 293T and RL95 cells expressing the sensors were implanted on either side of the animals hind limbs. The animals were repetitively imaged (with and without i.p. injection of $0.5 \mathrm{mg}$ of the ER ligand antagonist raloxifene) by injecting $3 \mathrm{mg}$ of the substrate D-luciferin. All mice $(\mathrm{n}=5$ each) were imaged using a cooled charge coupled device (CCD) camera (Xenogen IVIS; Xenogen Corp. Alameda, CA) and photons emitted from the mice were collected and integrated for a period of $1 \mathrm{~min}$. Images were analyzed using Living Image software (Xenogen) and Igor image analysis software (Wavemetric, OR). To quantify the number of emitted photons, regions of interest (ROI) were drawn over the area of the implanted cells and the maximum photons $/ \mathrm{sec} / \mathrm{cm}^{2} / \mathrm{steradian}(\mathrm{sr})$ were obtained as previously described ${ }^{17,} 19$. 


\section{Results}

Comparison of previously reported split renilla (Nrluc 229/Crluc 229) and split firefly luciferases (Nfluc 437/Cfluc 437 and Nfluc 416/Cfluc 398) using a fragment-assisted complementation strategy shows that the split renilla luciferase strategy has a higher fold induction with near zero background signal

The split renilla luciferase fragments identified from our previous study (Nrluc 229/Crluc $229)^{8}$, the split firefly luciferase fragments used by us in our previous studies (Nfluc $437 /$ Cfluc 437) $)^{7,11}$ and those used by others (Nfluc 416/Cfluc 398) ${ }^{12}$ were compared using vectors constructed to express the rapamycin mediated protein-protein (FRB/FKBP12) interaction system. All three split-reporter systems were studied in transiently co-transfected $293 \mathrm{~T}$ cells. The cells were assayed for luciferase activity before and 24 hours after exposure to $40 \mathrm{nM}$ of rapamycin. The results show relatively low levels of firefly luciferase activity before exposure to rapamycin and a $60 \pm 10$ fold $\left(6 \pm 2 \times 10^{4} \mathrm{RLU} / \mu \mathrm{g}\right.$ protein $\left./ \mathrm{min}\right)$ signal increase upon exposure to rapamycin from the combination of split firefly luciferase enzyme fragments (NFluc 437/CFluc 437) used previously by our labs. The split firefly luciferase enzyme fragments identified by others (NFluc 416/CFluc 398) ${ }^{12}$ show luciferase signal both in the presence and the absence of rapamycin, with a $6 \pm 2$ fold greater signal after exposure to rapamycin $\left(3.2 \pm 0.4 \times 10^{7} \mathrm{RLU} / \mu \mathrm{g}\right.$ protein/min) (Figure 1).

The split renilla luciferase system developed by us (NRluc 229/CRluc 229) shows a very low signal without rapamycin, however it achieves only 65 to $75 \%$ of the absolute signal $\left(2.2 \pm 0.3 \times 10^{7} \mathrm{RLU} / \mu \mathrm{g}\right.$ protein/min) in the presence of rapamycin achieved by the split firefly luciferase system identified by others ${ }^{12}$ (Figure 1 ). These results motivated us to develop an improved split firefly luciferase system that would have a relatively high absolute signal in the presence of rapamycin such as that seen with Nfluc 416/Cfluc 398, but without the high background signal (without rapamycin).

\section{Combinatorial screening with optical CCD camera imaging establishes that approximately $25 \%$ of the combinations studied show a significant level of firefly luciferase signal after exposure to rapamycin}

To identify $\mathrm{N}$ - and $\mathrm{C}$ - terminal firefly luciferase enzyme fragments that further improve the ability to study protein-protein and other interactions, 115 different combinations were screened by constructing vectors with rapamycin mediated interacting proteins FRB/ FKBP12. 293T cells co-transfected with different combinations in a 96 well format were imaged after $48 \mathrm{hrs}$ of exposure to rapamycin by optical CCD camera imaging by adding the substrate D-Luciferin to identify combinations of fragments that lead to luciferase signal through complementation. The results show bioluminescence signal from a significant number of combinations (29/115 or $25 \%$ ) only after exposure to rapamycin. Similarly, 3 out of 115 combinations $(2.6 \%)$ produce bioluminescence signal both in the presence and absence of rapamycin (self-complementation) (Supplementary Table 1; Supplementary Figure S1). From among the 29 combinations that generated rapamycin-mediated bioluminescence, 20 combinations with the highest signals were selected for further evaluation by luminometry.

Selected combinations identified by optical CCD camera imaging and evaluated further by luminometry show three split firefly luciferase combinations that are sensitive in producing a relatively high level of rapamycin mediated protein-protein interaction associated bioluminescence signal with an extremely low background signal

Twenty leading combinations of split $\mathrm{N}$ - and $\mathrm{C}$-terminal firefly luciferase fragment candidates were selected from an initial screen for further evaluation. These fragments were studied by luminometry in co-transfected $293 \mathrm{~T}$ cells with and without exposure to 
rapamycin. Three combinations of $\mathrm{N}$ - and $\mathrm{C}$ - terminal luciferase enzyme fragments produce a significant amount of rapamycin mediated luciferase signal (NFluc 398/CFluc 394: 800 \pm 150 fold with an absolute signal of $1.7 \pm 0.5 \times 10^{8} \mathrm{RLU} / \mu \mathrm{g}$ protein/min; NFluc 398/CFluc 398 : $2550 \pm 500$ fold with an absolute signal of $0.6 \pm 0.2 \times 10^{8} \mathrm{RLU} / \mu \mathrm{g}$ Protein/Min; NFluc 416/ CFluc 415: $250 \pm 60$ fold with an absolute signal of $1.6 \pm 0.4 \times 10^{8} \mathrm{RLU} / \mu \mathrm{g}$ Protein/Min) (Figure 2). Of these three, we selected Nfluc 398/Cfluc 394 for further experimentation because of its greater overall fold-induction and high absolute signal. Co-transfection experiments using RL95, MCF7 and MDA-MB-231 cells with all three fragment combinations show a similar pattern of results seen with the 293T cells (data not shown).

\section{Studying the orientation specific interaction of proteins and their associated reporter fragment complementation shows a preferred orientation for optimal signal production}

We next wanted to determine the optimal orientation of NFluc- and CFluc- reporter fragments and the interacting partners required for efficient protein-protein interaction mediated complementation. Eight different vectors were constructed to express fusion proteins with the reporter fragment and the interacting proteins in different orientations by using the most optimal reporter fragments Nfluc-398/Cfluc-394 and the previously published reporter fragments Nfluc-416/Cfluc-398 [Nfluc 416-FRB, Nfluc 398-FRB, FRBNfluc 416, FRB-Nfluc 398, Cfluc 398-FKBP12, Cfluc 394-FKBP12, FKBP12-Cfluc 398 and FKP12-Cfluc 394]. All these vectors in different combinations were studied in cotransfected 293T cells with and without rapamycin. The specific orientation of the fused reporter fragment/protein determines the level of signal produced. The fusion proteins NFluc 398-FRB/FKBP12-CFluc 394 generated $800 \pm 200$ fold induction with an absolute level of luciferase signal of $1.7 \pm 0.5 \times 10^{8} \mathrm{RLU} / \mu \mathrm{g}$ Protein/Min. Other combinations of the same expressed reporter fragment and interacting proteins, but in different orientations, (NFluc 398-FRB/CFluc 394-FKBP12: $20 \pm 4$ fold/1.5 $\pm 0.3 \times 10^{6} \mathrm{RLU} / \mu \mathrm{g}$ Protein/Min; FRB-NFluc 398/FKBP12- CFluc 394: 320 \pm 40 fold/0.8 $\pm 0.2 \times 10^{7}$ RLU/ $\mu$ g Protein/Min; FRB- NFluc 398/ CFluc 394-FKBP12: $2 \pm 1$ fold/ $0.3 \pm 0.2 \times 10^{6} \mathrm{RLU} / \mu \mathrm{g}$ Protein/Min) show lower levels of induction with different absolute levels of signal (Figure 3).

\section{Studying the optimal new combination of luciferase enzyme fragments with different interacting protein partners or intramolecular folding demonstrate robustness of the new split sites}

To evaluate the efficiency of the newly identified combination of $\mathrm{N}$ - and C- terminal luciferase enzyme fragments (NFluc 398/CFluc 394) with different interacting proteins; we constructed vectors expressing $\mathrm{N}$ - and $\mathrm{C}$-terminal fragments with four different interacting partners other than the one used for initial screening, with different types of interaction patterns include the (i) Id/myoD interaction system ${ }^{11}$, (ii) TK-TK- homodimerization system $^{20}$, (iii) ER-ligand induced homodimerization system (unpublished data) and (iv) Hypoxia induced interaction system (HIF1- $\alpha / \mathrm{pVHL}$ ) system (unpublished data), and the ERligand induced intramolecular folding system ${ }^{16}$. All these systems were studied in transiently transfected and co-transfected 293T cells under appropriate conditions. The results show significant levels of protein-protein interaction and intramolecular ER folding associated luciferase signal with expected specificity for all the systems using the newly identified NFluc 398/CFluc 394 fragments (Figure 4).

In the Id/myoD interaction system and the TK/TK homodimerization system, the expressed interaction proteins with the reporter protein fragments undergo protein-protein interaction mediated complementation and generate signal. So we modulated the level of protein by varying the concentration of DNA used for the study. These results show a protein concentration dependent increase in the complemented luciferase signal (Figure 4a, b). Whereas in the ER-ER homodimerization system and ER-ligand induced intramolecular 
folding systems, the binding of ER-ligands induces the level of luciferase fragments complementation. The result show specific ER-ligand induced ER-homodimerization and the ER-intramolecular folding and the subsequent complemented luciferase signal (Figure $4 \mathrm{c}$ and e). Similarly, in the hypoxia induced interaction system the level of protein hydroxylation controls the level of protein-protein interaction. This system shows efficient reduction in the complemented luciferase signal when the cells are exposed to hypoxic conditions (CoCl2 and DFO) that lead to reduction in the level of HIF1- $\alpha$ hydroxylation (Figure 4d).

\section{Optical CCD camera imaging of HIF1- $\alpha / p V H L$ interaction shows a hypoxia-induced reduction in complemented luciferase signal from cells}

To show that the signal level generated by the new combination of luciferase enzyme fragments was significant enough for imaging cells in culture and living animals, we selected a hypoxia inducible interaction system. The protein HIF1- $\alpha$ is phosphorylated by the enzyme prolyl hydroxylase in cells under normoxic conditions. The phosphorylated form of this protein interacts with the von Hippel-Lindau (pVHL) tumor suppressor protein. Hypoxic conditions lead to change in the phosphorylation level of HIF1- $\alpha$ which in turn reduces its interaction with pVHL. Exposures of cells to either $\mathrm{CoCl}_{2}$ or DFO have been reported to generate hypoxic conditions. Hence 293T cells co-transfected with vectors expressing fusion proteins NFluc 398-HIF1- $\alpha$ and pVHL-CFluc 394 were imaged after treatment with either $\mathrm{CoCl}_{2}$ or DFO for 24 hours. These results show a significant level of protein-protein interaction assisted luciferase signal as compared to the cells not exposed to either $\mathrm{CoCl}_{2}$ or DFO (normoxia). Cells exposed to either $\mathrm{CoCl}_{2}$ or DFO exhibit induction of hypoxia that in turn leads to a drop in protein-protein interaction and consequently a decrease in luciferase signal. The signal level decreases by $3 \pm 2$ fold with DFO, and $6 \pm 2$ fold with $\mathrm{CoCl}_{2}$ (Supplementary Figure S2).

\section{Imaging of ligand induced ER-intramolecular folding with the new combination of $\mathrm{N}$ - and C- terminal firefly luciferase enzyme fragments show a greater level of complemented luciferase signal upon induction by either the injected or endogenous ligand in living animals}

To check the efficiency of the newly identified firefly luciferase fragments in living animals, the ER-ligand induced intramolecular folding system was selected. We constructed three different sensor systems containing the estrogen receptor ligand binding domain (amino acids 281-595) and different combinations of firefly luciferase enzyme fragments as shown in Supplementary Figure 3a (NFluc 416, NFluc 398, CFluc 398, and CFluc 394). These three different sensors were transiently transfected using $293 \mathrm{~T}$ cells and studied by exposure of cells to different ER-ligands (Supplementary Figure S4). The results show efficient ligand induced intramolecular folding for all three sensors with different levels of absolute signal. The sensor constructed with $\mathrm{N}$ - and $\mathrm{C}$ - terminal luciferase fragments having a higher level of overlap [Sensor 3: NFluc 416/CFluc 394] shows significant level of background before the addition of ligands, and higher level of signal after introduction of ligand. The sensor constructed without any overlapping fragments [Sensor 1: NFluc 398/CFluc 398] shows very low background signal with highly specific ligand induced complementation signal. The sensor with the combination of newly identified fragments (sensor 2) shows very high ligand induced complementation signal with a low background signal

\section{Molecular imaging in living animals using the ER ligand induced intramolecular folding strategy with different sensors constructed using the new split sites demonstrates a high sensitivity for in vivo applications}

To demonstrate the use of newly identified fragments with improved sensitivity for applications in living animals; we selected the ER ligand induced intramolecular folding 
strategy. The 293T cells stably expressing sensors 2 and 3, and the RL95 cells stably expressing sensor 3 were used for the study (Supplementary Figure S3b). In a first set of experiment, male nude mice $(n=5)$ subcutaneously implanted with 5 million each of $293 \mathrm{~T}$ cells expressing sensors 2 and 3 were imaged for several days with and without the ligand antagonist raloxifene ( $20 \mu \mathrm{g}$ in $50 \mu \mathrm{l}$ sesame oil). The results show no significant signal from the implanted cells expressing the sensors. This is due to the unavailability of ER-ligands to induce complementation. When these animals received the ER-ligand raloxifene (Figure 5a/ Day 3), a significant level of complementation mediated luciferase signal is detected through the intramolecular folding induced by the ligand (Figure 5a/Day 4). Similarly in a second set of experiment, female nude mice implanted with 5 million each of $293 \mathrm{~T}$ and RL95 cells expressing sensors 2 and 3 respectively were imaged for several days without administering any ligands to observe the complementation induced by the endogenous estradiol that usually changes during different phases of the estrous cycle. The result shows estrous phase dependent complementation with a peak signal approximately once every 4-5 days (Figure 5b\&c). The absolute level of signals and the day at which these animals attained peak luciferase signals are different for each animal used for imaging. This reflects variations in the level of ligand produced by each animal.

\section{Discussion}

In this study we used a combinatorial screening approach to identify a new combination of N- (NFluc 398) and C- (CFluc 394) terminal firefly luciferase enzyme fragments that work efficiently in studying protein-protein interactions and intramolecular folding with a nearzero background signal (without producing self complementation associated signal). As we have been working for more than five years in developing split reporter based optical imaging techniques to study different cellular events, we have available to us several different combinations of split luciferase fragments with different complementation properties ${ }^{11}, 15$. We used all these fragments for this combinatorial screening study by constructing vectors expressing fusion proteins with different $\mathrm{N}$ - and $\mathrm{C}$ - terminal firefly luciferase fragments from the library and the rapamycin mediated interacting proteins FRB (FKBP12 Rapamycin Binding domain) and FKBP12 (FK506 Binding protein). The rapamycin-mediated interacting proteins FRB and FKBP12 were selected for this study because in a single step it is possible to distinguish between signals generated from selfcomplementing reporter fragments, and through protein-protein interaction assisted reporter fragment complementation, using high-throughput optical CCD camera imaging.

Although we have previously reported split renilla luciferase fragments with near-zero background signal for studying protein-protein interactions, the blue-green emission spectrum of renilla luciferase (480-510 nM) penetrates tissues less efficiently, sometimes limiting its sensitivity in some small living animal applications ${ }^{12,17,18}$. The split firefly luciferase fragments (Nfluc 437/Cfluc 437) used in our previous study have limited signal thereby precluding their generalizability. The split firefly luciferase enzyme fragments used by others (Nfluc 416/Cfluc 398) show a relatively high level of signal before protein-protein interactions occur, leading to potential difficulty in differentiating from the low level of protein-protein interaction mediated signal generated from weak interacting partners ${ }^{12}$. Hence the combination of fragments identified in this study (Nfluc 398/Cfluc 394) and evaluated using several different protein partners and an intramolecular folding strategy overcome many of the problems encountered from previously reported systems. Even though the identified fragments of this study are more optimal than any previous ones, future studies may still identify better split reporter combinations.

In addition, in this study we also evaluated the relative orientation between the interacting protein partner and the split reporter that leads to optimal PFAC. Different studies from our 
lab have found that attaching small peptides or proteins of different lengths to the $\mathrm{NH}_{2}$ terminus of the firefly luciferase protein significantly reduces the luciferase enzyme activity $^{21}$. The study by Luker et al ${ }^{12}$ achieved a significant level of complemented luciferase signal by attaching the protein FRB to the $\mathrm{NH}_{2}$ terminus of the Nfluc fragment. Hence in this study to further confirm the orientation that is essential for efficient PFAC, we constructed several different vectors expressing all the different possible orientations by using selective $\mathrm{N}$ - and $\mathrm{C}$ - terminal luciferase enzyme fragments. The preferred orientation identified from this study is Nfluc-FRB/FKBP12-CFluc. We also found that the newly identified fragments showed consistent result across several cell lines used for the study. In addition the newly identified fragments showed similar level of sensitivity to the split renilla luciferase fragments, without resulting in any steric hindrance for the estrogen receptor intramolecular folding system.

The ER-ligand induced intramolecular folding system utilizing the newly identified luciferase fragments was used to evaluate the complementation induced by the injected ligand antagonist (raloxifene) in living mice. Similarly by using this system we also estimated the level of complementation induced by circulating endogenous ligand in female nude mice. This study also identified many different combinations of non-overlapping and overlapping luciferase fragments that can be used for studying different cellular events such as sub cellular localization of proteins, cell-cell fusion, and evaluating cell delivery vehicles where self-complementing split reporters are needed.

In summary, in this study we compared previously published split sites for firefly and renilla luciferase. We sought to overcome limitations of previous split reporters and used a combinatorial approach to identify a new split site for firefly luciferase with optimal characteristics. We tested this new split site with several different interacting proteins and with an intramolecular folding strategy in cell culture. Optical imaging in small living animals further demonstrates the utility of the new split sites. This new split reporter complementation system with greater absolute signal and lower background than previous systems can be further extended to study PFAC and other intracellular interactions. The developed system can also be used for high-throughput screening of new protein-protein interaction targeted drugs in cells along with further evaluation in small living animals.

\section{Supplementary Material}

Refer to Web version on PubMed Central for supplementary material.

\section{Acknowledgments}

This work is supported in part by NIH grants, R01 CA82214 (SSG), ICMIC P50 CA114747 (SSG), and the Small Animal Imaging Resource Program (SAIRP).

\section{Abbreviations}

$\begin{array}{ll}\text { Fluc } & \text { Firefly luciferase protein } \\ \text { fluc } & \text { Firefly luciferase gene } \\ \text { RLuc } & \text { Renilla luciferase protein } \\ \text { rluc } & \text { Renilla luciferase gene } \\ \text { FKBP12 } & \text { FK506 binding protein } \\ \text { FRB } & \text { FKBP12-rapamycin-binding domain }\end{array}$


ER estrogen receptor

LBD ligand binding domain

\section{References}

1. Paulmurugan R, Ray P, De A, Chan CT, Gambhir SS. CSHL. 2005; Chapter 35

2. Paulmurugan R, Ray P, De A, Chan CT, Gambhir SS. TrAC Trends in Analytical Chemistry. 2005; 24:446-458.

3. Pelletier JN, Arndt KM, Pluckthun A, Michnick SW. Nat Biotechnol. 1999; 17:683-690. [PubMed: 10404162]

4. Pelletier JN, Campbell-Valois FX, Michnick SW. Proc Natl Acad Sci U S A. 1998; 95:1214112146. [PubMed: 9770453]

5. Wehrman T, Kleaveland B, Her JH, Balint RF, Blau HM. Proc Natl Acad Sci U S A. 2002; 99:3469-3474. [PubMed: 11904411]

6. Ghosh I, Hamilton AD, Regan L. J Am Chem Soc. 2000; 122:5658-5659.

7. Ozawa T, Kaihara A, Sato M, Tachihara K, Umezawa Y. Anal Chem. 2001; 73:2516-2521. [PubMed: 11403293]

8. Paulmurugan R, Gambhir SS. Anal Chem. 2003; 75:1584-1589. [PubMed: 12705589]

9. Luker GD, Sharma V, Pica CM, Dahlheimer JL, Li W, Ochesky J, Ryan CE, Piwnica-Worms H, Piwnica-Worms D. Proc Natl Acad Sci U S A. 2002; 99:6961-6966. [PubMed: 11997447]

10. Ray P, Pimenta H, Paulmurugan R, Berger F, Phelps ME, Iyer M, Gambhir SS. Proc Natl Acad Sci U S A. 2002; 99:3105-3110. [PubMed: 11854471]

11. Paulmurugan R, Umezawa Y, Gambhir SS. Proc Natl Acad Sci U S A. 2002; 99:15608-15613. [PubMed: 12438689]

12. Luker KE, Smith MC, Luker GD, Gammon ST, Piwnica-Worms H, Piwnica-Worms D. Proc Natl Acad Sci U S A. 2004; 101:12288-12293. [PubMed: 15284440]

13. Contag CH, Bachmann MH. Annu Rev Biomed Eng. 2002; 4:235-260. [PubMed: 12117758]

14. Kaihara A, Kawai Y, Sato M, Ozawa T, Umezawa Y. Anal Chem. 2003; 75:4176-4181. [PubMed: 14632132]

15. Paulmurugan R, Gambhir SS. Anal Chem. 2005; 77:1295-1302. [PubMed: 15732910]

16. Paulmurugan R, Gambhir SS. Proc Natl Acad Sci U S A. 2006; 103:155883.

17. Paulmurugan R, Massoud TF, Huang J, Gambhir SS. Cancer Res. 2004; 64:2113-2119. [PubMed: 15026351]

18. Paulmurugan R, Gambhir SS. Cancer Res. 2005; 65:7413-7420. [PubMed: 16103094]

19. Bhaumik S, Gambhir SS. Proc Natl Acad Sci U S A. 2002; 99:377-382. [PubMed: 11752410]

20. Massoud TF, Paulmurugan R, Gambhir SS. Faseb J. 2004; 18:1105-1107. [PubMed: 15132989]

21. Ray P, De A, Min JJ, Tsien RY, Gambhir SS. Cancer Res. 2004; 64:1323-1330. [PubMed: 14973078] 


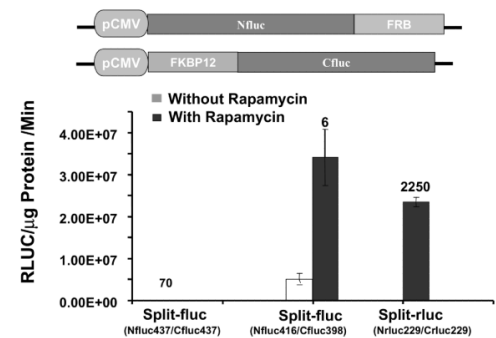

Figure 1.

General scheme of different vectors with interacting proteins constructed for studying combinatorial screening. Comparison of different bioluminescent optical reporters in fragment-assisted complementation systems with rapamycin mediated heterodimerization proteins. The 293T cells co-transfected with (1) Nfluc 437-FRB/FKBP12-Cfluc 437, (2) Nfluc 416-FRB/FKBP12-Cfluc 398 and (3) Nrluc-FRB/FKBP12-Crluc assayed for luciferase activity with and without exposure to rapamycin. The results show significant $(p<0.05)$ level of rapamycin mediated luciferase activity from all three systems. 


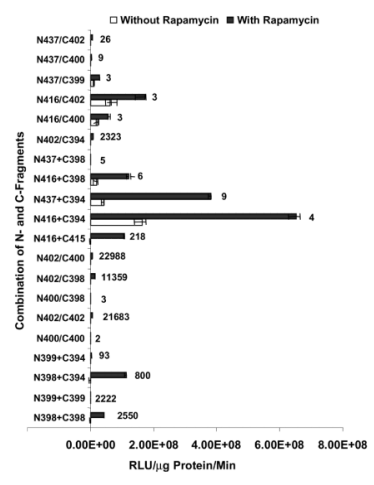

Figure 2.

Luminometer assay results from the lysates of $293 \mathrm{~T}$ cells co-transfected with selected combinations identified by optical CCD camera imaging shown in supplementary figure 1. The cells were assayed with and without exposure to rapamycin. The results show a significant level of rapamycin mediated protein-protein interaction assisted luciferase signal from several combinations, and identify a particular combination (Nfluc 398/Cfluc 394) with a very high level of protein-protein interaction signal and near-zero self-complementing background signal. 


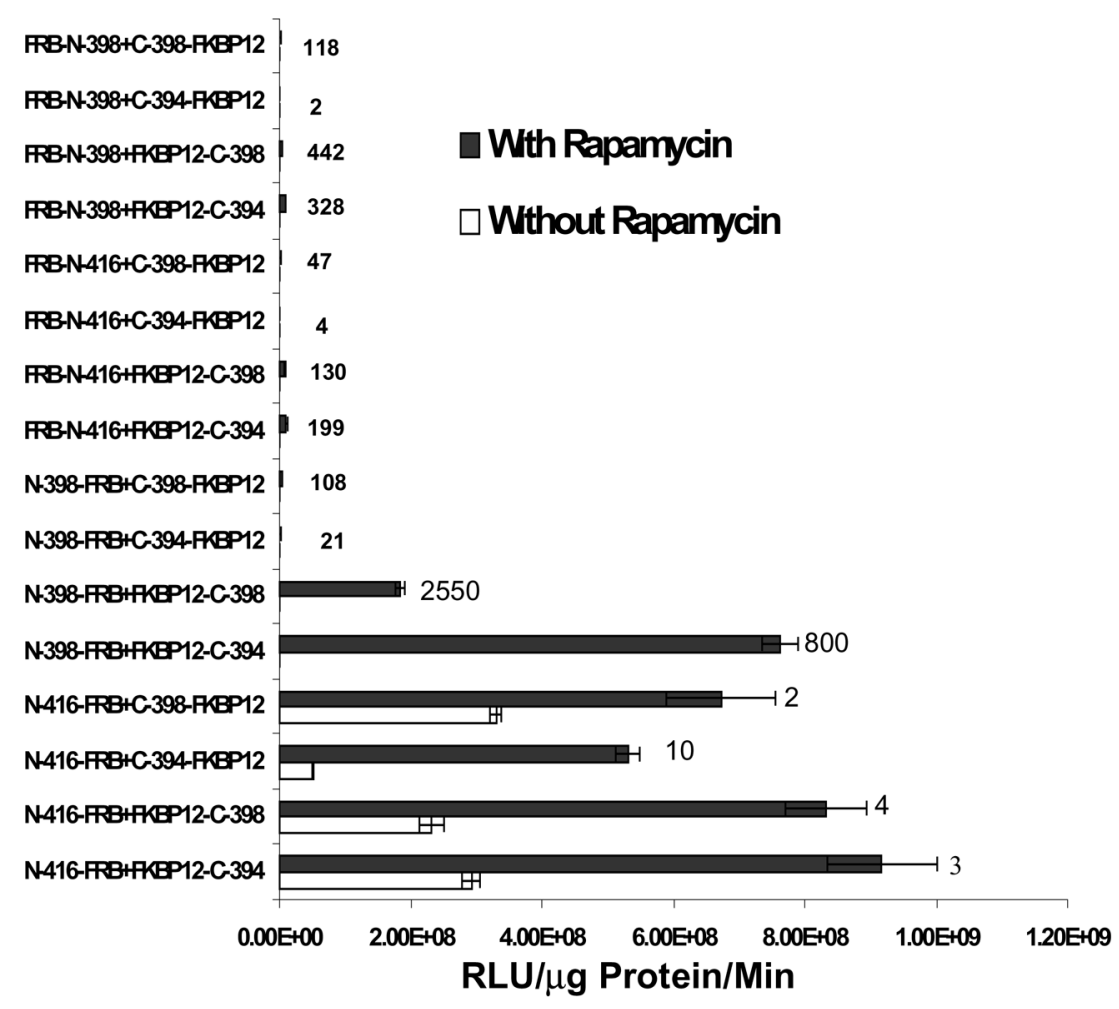

Figure 3.

Luminometer assay results from the lysates of $293 \mathrm{~T}$ cells co-transfected with different combinations of $\mathrm{N}$ - and $\mathrm{C}$ - terminal firefly luciferase fragments with interacting proteins FRB and FKBP12 constructed with different orientations. Orientation of an interacting protein with the reporter fragments is important for achieving efficient complementation. The combination containing NFluc-FRB/FKBP12-CFluc produced a greater level of rapamycin mediated protein-protein interaction signal over other orientations. 


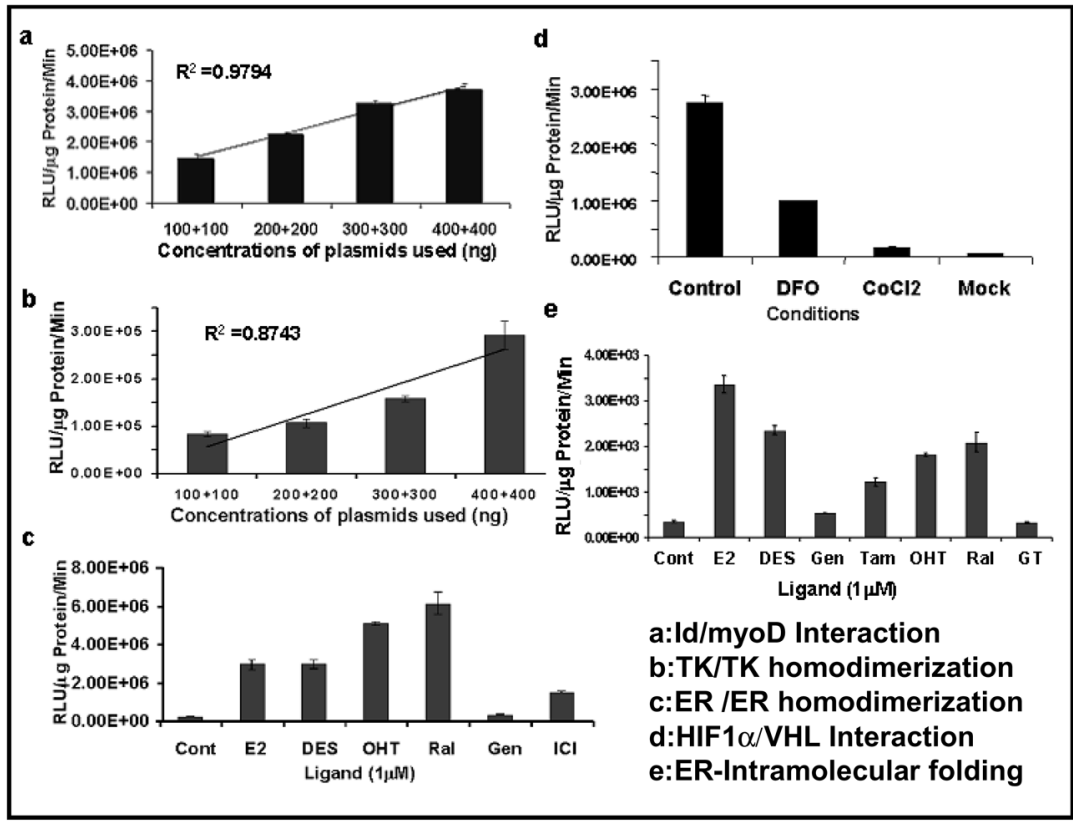

Figure 4.

Efficiency of newly identified combination of firefly luciferase fragments (NFluc 398/CFluc 394) with different interacting protein partners. $293 \mathrm{~T}$ cells co-transfected with vectors constructed to express the reporter fragments with interacting proteins (a) Id/myoD, (b) TKTK- homodimerization, (c) ER-ligand induced homodimerization, (d) Hypoxia induced interacting proteins (HIF1- $\alpha / \mathrm{pVHL}$ ) and (e) ER-ligand induced intramolecular folding were studied under appropriate conditions. A significant level of protein-protein interaction associated luciferase signal is seen with expected specificity from all the different systems used. 


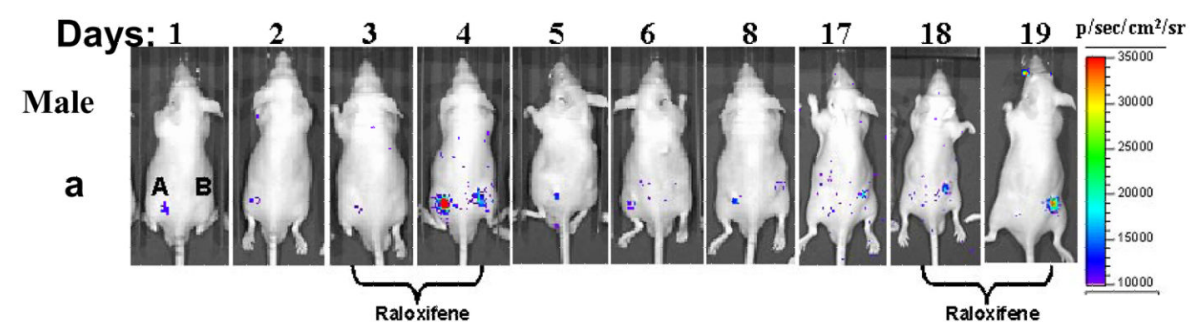

A: 293T-stable with sensor -2

B: 293T-stable with sensor -3

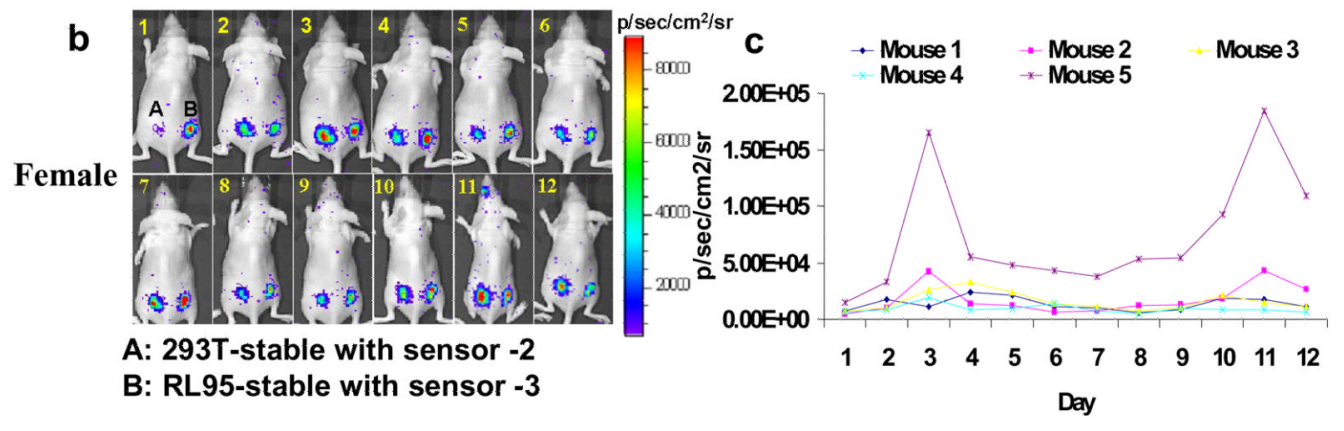

Fig. 5. Bioluminescence imaging sensors for measuring changes in endogenous estradiol levels in living mice

To study changes in endogenous estradiol levels in living mice three different sensors with split firefly luciferase enzyme fragments were constructed for making stable 293T and RL95 cells [Sensor 2. NFluc 398-hER-LBDF-CFluc 394): Generates low background signal with high complementation signal and Sensor 3. NFluc 416-hER-LBDF-CFluc 394): Generates high background with high complementation signal. 293T and RL95 cells stably expressing these sensors were used for repetitive non-invasive imaging of ER-intramolecular folding induced by endogenous estradiol in male (a) $(n=5)$ and female $(\mathbf{b})(n=5)$ mice. c. The bioluminescence signal was measured from five female mice for 12 days. The male mice show no significant sensor signal because of low levels of circulating estradiol. However in the presence of Raloxifene (days 4 and 19), there is signal as expected due to intramolecular ER folding. The female mice produce significant amounts of endogenous estradiol that fluctuates daily and this is reflected by the complementation signal produced from the sensors. 